\title{
Structure of Cellulose
}

$\mathrm{T}$ HE recent annual Convention of the Technical Section of the Paper Makers' Association of Great Britain was unusual in that the subject chosen was one which appeared at first sight to be more of academic than of practical interest. Fortunately, in the able hands of Prof. H. Mark, of the University of Vienna, who opened the conference, the subject lost many of its complexities, and technical men present were stimulated to attempt to apply theory to their practical problems.

Prof. Mark's paper, which is published in a special issue of the World's Paper Trade Review, dealt principally with the structure of cellulose. He supported the theory, now generally accepted, that cellulose is built up of so-called micelles consisting of bundles of parallel chains of glucose molecules, and he dwelt at some length on the controversy which at present concerns the actual length of these chains. The majority of the evidence, including that based on the chemical properties of cellulose, points to a length of 150-200 glucose units, and yet one cannot ignore the work of Staudinger on viscosity, which can only be explained by a much higher figure. It may be that the lower figure represents a minimum value.

In opening the general discussion, Sir William Bragg referred to the X-ray evidence on which so much of this work is based. Subsequent speakers paid some attention to the much-debated question of the mechanism of the hydration which occurs when paper is beaten, and a suggestion was made that the resulting change in structure is analogous to that which occurs in the manufacture of true cellulose plastics by chemical reaction.

Prof. Mark also indicated how such theories might be applied to problems in the art-silk industry, although it seems that they can do little at present to explain what occurs when cellulose is beaten for paper-making, as this problem is concerned with water absorption rather than with modification of crystalline structure. When, for example, a silk fibre is bent, the outer layers are stressed more than the inner core, and so long as the elastic limit of the latter is not surpassed the fibre will return to its original position and will thus show resistance to creasing. Unfortunately, this resistance is usually decreased when a fibre is treated so as to increase its tensile strength, but it is now possible to obtain the core more highly orientated than the surface and so to preserve both properties. Here one can take a leaf from the book of Nature, since this difference in orientation is already present in the natural silk fibre. If, however, the silkworm is placed on a revolving rod, and is therefore compelled to spin more rapidly, it descends to the level of human endeavour so far as lack of creasing-resistance of its product is concerned.

Prof. Mark obviously voiced the opinion of those present when he stated that the interaction of water and cellulose is one of the most interesting questions in paper-making. He chose to approach the problem by taking absorption capacity as an index of changes produced in the area and quality of the surface of the fibre by chemical treatment or by beating. His results indicate that hydroxyl groups are responsible for the fixation of water molecules on the surface of the cellulose, and act by virtue of the secondary valencies of the oxygen atoms; treatment with a chemical reagent opens up small slits or holes in the micelle into which further molecules of water may pass. Penetrations of solvents and swelling agents into the cellulose lattice is not unknown, but as a rule X-rays show evidence of well-defined addition compounds. Until now, however, no one has succeeded in preparing a true cellulose hydrate, so that an important link in an otherwise strong chain of evidence is still missing.

\section{Sunspots and Meteorological Phenomena}

$\mathrm{I}^{\mathrm{N}}$ a monograph entitled "L'Influence Solaire et les Progrès de la Météorologie"* Henri Memery advances the theory that sunspots show a tendency to increase and decrease at certain definite times of the year, and that this tendency is shown whatever period of years is considered. He suggests further that there is a relationship between the amount of sunspot development and numerous terrestrial disturbances, for example, spells of abnormal rainfall and temperature, and also between individual groups of sunspots and meteorological phenomena. Fifty years of solar and meteorological observations made at the Observatoire de Physique solaire et de Météorologie, Talence, provide a large part of the experimental data on which his views are based.

To most astronomers and meteorologists, the theory that sunspot activity shows any permanent relationship with the dates on our terrestrial calendar will probably appear a highly improbable one, to be accepted only in the face of overwhelming evidence of

- I'Influence solaire et les progrès de la météorologie: résultats de 50 années d'observations solaires et météorologiques comprenant les observations et les recherches effectuées à Talence à partir de 1900 Par Henri Mémery. Pp.iv + 23. (Talence : Observatoire de Physique solaire et de Météorologie, 1932. its truth. The evidence in this paper rests upon curves showing the average area on the sun's disc covered by sunspots on each day of the year for periods of 12,41 and 50 years. There are, however, apparent discrepancies between the lengths of the periods implied by the statements made alongside these curves and those given above the curves. The periods are quoted as 12,40 and 50 years in the latter case and it is possible that 40 should replace 41 in the algebraic expressions given below. It will be seen, however, that the conclusions reached are not affected by this uncertainty, nor even by the doubt as to whether the 12 year period should not have been taken as 13 years. Few would deny that the three curves show considerable similarity, but the periods covered are 1889-1901, 1880-1920, and $1880-1929$, that is, the 12 years in the first group are used also in the groups for 41 and 50 years, and the overlap is even greater for the 41 and 50 year means.

The question arises whether the similarity in the curves cannot be explained simply by this overlap. Take the case of the curves for 12 and 41 year periods. The similarity between these is held to imply a positive correlation between the ordinates. The two 